\title{
GROUPES DE LIE PSEUDO-RIEMANNIENS PLATS
}

\author{
Anne Aubert et Alberto Medina
}

(Received October 11, 2001, revised September 19, 2002)

\begin{abstract}
The determination of affine Lie groups (i.e., which carry a left-invariant affine structure) is an open problem ([12]). In this work we begin the study of Lie groups with a left-invariant, flat pseudo-Riemannian metric (flat pseudo-Riemannian groups). We show that in such groups the left-invariant affine structure defined by the Levi-Civita connection is geodesically complete if and only if the group is unimodular. We also show that the cotangent manifold of an affine Lie group is endowed with an affine Lie group structure and a leftinvariant, flat hyperbolic metric. We describe a double extension process which allows us to construct all nilpotent, flat Lorentzian groups. We give examples and prove that the only Heisenberg group which carries a left invariant, flat pseudo-Riemannian metric is the three dimensional one.
\end{abstract}

Introduction et Résumé. Un groupe de Lie pseudo-riemannien plat $G$ est un groupe de Lie muni d'une métrique invariante à gauche plate pseudo-riemannienne. L'algèbre de Lie d'un tel groupe sera dite pseudo-métrique plate. La connexion de Levi-Civita de $G$ fait de $G$ un groupe de Lie affine, c'est-à-dire à structure affine invariante à gauche. Seuls les groupes de Lie riemanniens plats sont connus. Ils ont été décrits par Milnor dans ([11], p. 298).

Etant données une structure affine et une métrique pseudo-riemannienne $g$ invariantes à gauche sur $G$, on dit que $g$ est localement hessienne si elle s'exprime en coordonnées affines comme la hessienne d'une fonction. Si $g$ est plate, un argument identique à celui de Shima ([14], p. 213) prouve que $g$ est localement hessienne relativement à la structure affine associée.

Voici une description succinte de nos principaux résultats concernant les groupes de Lie pseudo-riemanniens plats et (ou) leurs algèbres.

La structure affine est géodésiquement complète si et seulement si le groupe est unimodulaire (Théorème 1.1).

La variété cotangente d'un groupe de Lie affine est munie d'une structure de groupe de Lie à métrique plate hyperbolique, c'est-à-dire de signature $(n, n)$, invariante à gauche canonique (Section 2).

Les groupes de Lie nilpotents lorentziens plats sont obtenus par un procédé de double extension d'un groupe de Lie riemannien plat abélien par un groupe de Lie unidimensionnel (Théorème 3.1).

Notre notion de double extension d'une algèbre pseudo-métrique plate par une droite (Section 3) est analogue à la notion de double extension des algèbres de Lie symplectiques ou orthogonales de Medina-Revoy ([9], [10]).

2000 Mathematics Subject Classification. Primary 53C50; Secondary 22 E60.

Key words and phrases. Flat pseudo-Riemannian Lie groups, affine Lie groups, geodesic completeness. 
Les algèbres de Lie des groupes de Lie pseudo-riemanniens plats dont la connexion de Levi-Civita est bi-invariante s'obtiennent par une suite de doubles extensions par une droite à partir de l'algèbre à métrique plate de dimension un ou zéro (Proposition 3.3).

La notion de double extension permet de prouver que le seul groupe d'Heisenberg qui soit muni d'une métrique pseudo-riemannienne plate invariante à gauche est celui de dimension 3. Dans ce cas, la métrique est lorentzienne (Proposition 4.1).

Dans Section 2, nous caractérisons les groupes de Lie à métrique hyperbolique plate invariante à gauche contenant un sous-groupe de Lie distingué commutatif lagrangien, c'està-dire totalement isotrope de dimension la moitié de la dimension du groupe (Théorème 2.1).

Malgré le fait que $G L(n, \boldsymbol{R})$ ait des pseudo-métriques hessiennes bi-invariantes relatives à des structures affines bi-invariantes, il n'admet pas en général de métrique pseudoriemannienne plate invariante à gauche (Théorème 4.1).

Les auteurs remercient M. Bordemann pour ses remarques utiles, en particulier celles concernant Lemme 1.1.

Dans la suite, les groupes de Lie seront supposés connexes, et par objets invariants sur des groupes de Lie nous entendrons objets invariants par les translations à gauche du groupe.

1. Groupes de Lie pseudo-riemanniens plats et groupes de Lie affines. Soit $G$ un groupe de Lie muni d'une métrique pseudo-riemannienne plate invariante $\mu$. La connexion de Levi-Civita $\nabla$ associée à $\mu$ étant plate, elle définit une structure affine invariante à gauche sur $G$. D'autre part, puisque pour tous $X, Y, Z$ champs de vecteurs sur $G$,

$$
\left.X \cdot \mu(Y, Z)=\mu\left(\nabla_{X} Y, Z\right)\right)+\mu\left(Y, \nabla_{X} Z\right),
$$

il résulte que l'on a pour tous $x, y, z$ dans $\mathcal{G}=T_{\varepsilon} G$, où $\varepsilon$ est l'élément neutre de $G$,

$$
x^{+} \cdot \mu\left(y^{+}, z^{+}\right)=\mu\left(\nabla_{x^{+}} y^{+}, z^{+}\right)+\mu\left(y^{+}, \nabla_{x} z^{+}\right),
$$

$x^{+}$étant le champ invariant à gauche associé à $x$. Ainsi, le produit, dit de Levi-Civita, défini $\operatorname{sur} \mathcal{G} \operatorname{par} x \cdot y=\left(\nabla_{x}+y^{+}\right)(\varepsilon)$ vérifie

$$
0=\langle x \cdot y, z\rangle+\langle y, x \cdot z\rangle
$$

où $\langle\rangle=,\mu_{\varepsilon}$.

Que la connexion $\nabla$ soit à courbure et torsion nulles s'exprime par les identités

$$
(x \cdot y) \cdot z-x \cdot(y \cdot z)=(y \cdot x) \cdot z-y \cdot(x \cdot z)
$$

$$
[x, y]=x \cdot y-y \cdot x
$$

pour tous $x, y, z$ dans $\mathcal{G}$.

Les formules (1) et (3) impliquent l'identité

$$
\langle x \cdot y, z\rangle=\frac{1}{2}\{\langle[x, y], z\rangle-\langle[y, z], x\rangle+\langle[z, x], y\rangle\} .
$$


Un espace vectoriel muni d'un produit $x \cdot y=L_{x} y=R_{y} x$ vérifiant (2) est appelé une algèbre symétrique à gauche (S.G). Dans une telle algèbre, le crochet défini par (3) est un crochet de Lie, dit sous-jacent au produit S.G. Si ce crochet coïncide avec un crochet de Lie donné au départ, on dit que le produit symétrique à gauche est compatible avec la structure de Lie. Ceci motive la notion suivante.

DÉFINITION 1.1. On appelle algèbre de Lie pseudo-métrique plate une algèbre de Lie $\mathcal{G}$ munie d'une forme quadratique non dégénérée $q$ et d'un produit symétrique à gauche compatible avec la structure de Lie tels que les multiplications gauches soient des endomorphismes de $\mathcal{G}$ antisymétriques par rapport à $q$.

Dans la suite, on appelera espace quadratique un espace vectoriel $V$ muni d'une forme quadratique non dégénérée $q$. Le groupe $I(V, q)$ des isométries de $(V, q)$ peut être considéré comme le produit semi-direct $(V,+) \times$ Id $O(V, q)$ du sous-groupe distingué $(V,+)$ de translations par le sous-groupe $O(V, q)$ de transformations orthogonales au moyen de la représentation identité. Son algèbre de Lie $V \times$ id $o(V, q)$ est donc le produit semi-direct de l'idéal abélien $V$ par la sous-algèbre $o(V, q)$ des isométries infinitésimales de $(V, q)$.

Pour commencer, remarquons le fait suivant.

Proposition 1.1. Soit G un groupe de Lie de groupe revêtement universel $\tilde{G}$. Alors $G$ est pseudo-riemannien plat si et seulement si $\tilde{G}$ admet une représentation affine par des isométries d'un espace quadratique $(V, q)$ ayant un point à orbite ouverte et isotropie discrète.

DÉmonstration. D'après les formules (1), (2) et (3), si $(G, \mu)$ est pseudoriemannien plat, l'application $\theta: x \mapsto\left(x, L_{x}\right)$ est un homomorphisme de Lie de $\mathcal{G}$ dans $\mathcal{G} \times$ id $o(\mathcal{G},\langle\rangle$,$) . Par passage à l'exponentielle, on obtient alors un homomorphisme de$ groupes de Lie

$$
\begin{aligned}
\rho: \tilde{G} & \rightarrow I(\mathcal{G}, q) \\
\sigma & \mapsto\left(Q(\sigma), F_{\sigma}\right) .
\end{aligned}
$$

Il est clair que l'application orbitale

$$
\begin{aligned}
\pi: \tilde{G} & \rightarrow \operatorname{Orb}(0) \\
\sigma & \mapsto Q(\sigma)+F_{\sigma}(0)
\end{aligned}
$$

est un revêtement.

Réciproquement, soient $\rho: \tilde{G} \rightarrow I(V, q)$ un homomorphisme de groupes de Lie et $v \in V$ un point tel que l'application orbitale $\pi: \tilde{G} \rightarrow \operatorname{Orb}(v)$ soit un revêtement. Munissons l'ouvert $\operatorname{Orb}(v)$ de $V$ de la connexion induite par la connexion usuelle $\nabla_{0}$ de $V$. Il est évident que $\nabla_{0}$ est la connexion de Levi-Civita de $q$. Puisque $\pi$ est équivariante par les actions de $\tilde{G}$ sur lui-même par multiplications gauches et sur $V$ moyennant la représentation $\rho$, la connexion $\nabla$ pré-image de $\nabla_{0}$ par $\pi$ est plate et invariante à gauche. En outre, puisque $\tilde{G}$ 
opère sur $(V, q)$ par des isométries, $\nabla$ est aussi la connexion de Levi-Civita associée à la pseudo-métrique invariante sur $\tilde{G}$ définie par $q$.

Voici une conséquence de la proposition.

THÉORÈmE 1.1. Soit $(G, \mu)$ un groupe de Lie pseudo-riemannien plat. La structure affine définie par la connexion de Levi-Civita est géodésiquement complète si et seulement si $G$ est unimodulaire.

DÉmonstration. En effet soit $x \in \mathcal{G}$ et $L_{x}^{*}$ le transposé de $L_{x}$ relativement à $\langle$,$\rangle .$ Soit $\varphi: \mathcal{G} \rightarrow \mathcal{G}^{*}$ l'isomorphisme canonique d'espaces vectoriels $(x \mapsto\langle x, \cdot\rangle)$. Puisque $L_{x}^{*}=\varphi^{-1} \circ{ }^{t} L_{x} \circ \varphi$, nous avons $\operatorname{tr}\left(L_{x}^{*}\right)=\operatorname{tr}\left({ }^{t} L_{x}\right)=\operatorname{tr}\left(L_{x}\right)$. Ceci prouve que $\operatorname{tr}\left(L_{x}\right)=0$, car nous savons que $L_{x} \in o(\mathcal{G},\langle\rangle$,$) , c'est-à-dire L_{x}+L_{x}^{*}=0$.

D'autre part, que $\nabla$ est géodésiquement complète signifie que les multiplications droites $y \mapsto R_{x} y=y \cdot x$ sont nilpotentes pour tout $x$ dans $\mathcal{G}$, ce qui revient à dire que l'homomorphisme d'algèbres de Lie $x \mapsto \operatorname{tr}\left(R_{x}\right)$ est nul ([6]). Or l'égalité $R_{x}-L_{x}=\mathrm{ad}_{x}$ nous permet de conclure que $\operatorname{tr}\left(R_{x}\right)=\operatorname{tr}\left(\operatorname{ad}_{x}\right)$.

Par conséquent pour que $\operatorname{tr}\left(R_{x}\right)=0$ pour tout $x$ dans $\mathcal{G}$, il faut et il suffit que $\operatorname{tr}\left(\operatorname{ad}_{x}\right)=0$ quel que soit $x$ dans $\mathcal{G}$, ce qui prouve le résultat.

La proposition permet de bien comprendre la preuve du résultat suivant.

THÉORÈME 1.2 (Milnor, [11]). Un groupe de Lie à métrique riemannienne invariante à gauche est plat si et seulement si son algèbre de Lie se décompose en une somme directe orthogonale $\mathcal{B} \oplus \mathcal{U}$, où $\mathcal{B}$ est une sous-algèbre abélienne, $\mathcal{U}$ un idéal abélien et $\mathcal{B}$ opère sur $\mathcal{U}$ par des transformations antisymétriques, c'est-à-dire $\operatorname{ad}_{b}$ appartient à $o(\mathcal{U},\langle\rangle$,$) , pour tout$ $b$ dans $\mathcal{B}$.

DÉMONSTRATION. L'idée de la preuve est la suivante. On considère la représentation d'algèbre de Lie $L$. Son noyau est un idéal bilatère d'algèbre symétrique à gauche car $R_{x}=$ $-\operatorname{ad}_{x}$ pour tout $x$ dans $\operatorname{Ker} L$. L'algèbre symétrique à gauche $\mathcal{G} / \operatorname{Ker} L$ est alors isomorphe à la sous-algèbre $\operatorname{Im} L$ de l'algèbre de Lie compacte $o(\mathcal{G}, q)$. Ainsi $\operatorname{Im} L$ se décompose en produit direct orthogonal d'algèbres de Lie simples ou de dimension 1. Puisque chacun de ses facteurs est symétrique à gauche, il n'y a pas de facteur simple, de sorte que $\operatorname{Im} L$ est abélienne. L'algèbre de Lie $\mathcal{G} / \operatorname{Ker} L$ étant isomorphe à $(\operatorname{Ker} L)^{\perp}$, il résulte que $\mathcal{G}$ est le produit semidirect $\operatorname{Ker} L \oplus(\operatorname{Ker} L)^{\perp}$, l'action de $(\operatorname{Ker} L)^{\perp}$ sur $\operatorname{Ker} L$ se faisant par des endomorphismes antisymétriques.

D'après la preuve de la proposition 1.1, il est évident qu'un groupe de Lie simplement connexe affine peut être regardé comme un sous-groupe du groupe des transformations affines de $\mathcal{G}=L(G)$. La clé de voûte du théorème de structure de Milnor réside dans le fait que $G$ admet des sous-groupes non triviaux formés de translations. Cette observation motive le résultat suivant, qui nous permettra de décrire la structure des groupes de Lie nilpotents lorentziens plats (Théorème 3.1). 
LEMME 1.1. Un groupe de Lie nilpotent lorentzien plat simplement connexe, vu comme groupe de transformations affines de son algèbre de Lie, contient des sous-groupes à un paramètre non triviaux formés de translations.

DÉmonstration. Soit $(G, g)$ un groupe de Lie nilpotent lorentzien plat simplement connexe d'algèbre de Lie $A$. Soient $\langle$,$\rangle la métrique lorentzienne plate sur A$ qui coïncide avec $g$ en l'élément neutre de $G$, et $(a, b) \mapsto a \cdot b=L_{a} b$ le produit de Levi-Civita associé.

D'après Proposition 1.1, le groupe $G$ admet une représentation affine $\rho$ par des isométries de l'espace quadratique $(A,\langle\rangle$,$) . Puisque l'on a$

$$
\rho(\exp a)=\exp \left(a, L_{a}\right)=\left(\sum_{k=1}^{\infty} \frac{1}{k !} L_{a}^{k-1}(a), \operatorname{Exp}_{a}\right)
$$

un élément non nul $a$ de $A$ tel que $L_{a}=0$ détermine un sous-groupe à un paramètre $(t \mapsto$ exp ta) non trivial formé de translations. Nous allons prouver que de tels éléments existent, c'est à dire que $N(A)=\left\{a \in A \mid L_{a}=0\right\}$ est non réduit à 0 .

Si $n+1$ est la dimension de $A$, l'application $L: a \mapsto L_{a}$ est un homomorphisme d'algèbres de Lie de $A$ dans $o(n, 1)$. Comme $G$ est nilpotent, $G$ est unimodulaire donc d'aprés Théorème 1.1, l'algèbre symétrique à gauche $A$ est nilpotente. Du fait que $A$ est nilpotente en tant qu'algèbre de Lie et en tant qu'algèbre symétrique à gauche, il suit d'aprés Proposition 26 de [6] que les $L_{a}$ sont nilpotents.

D'autre part, la sous-algèbre de Lie $o(n)$ de $o(n, 1)$ est compacte pour $n \geq 3$. Soit $L_{a}$ élément de la sous-algèbre $B:=\left\{L_{a}, a \in A\right\} \cap o(n)$ de $o(n, 1)$. Il existe alors un sous-espace $V$ de $A$ de dimension $n$ stable par $L_{a}$ tel que $\langle,\rangle_{\mid V \times V}$ soit définie positive et telle que $L_{a \mid V}$ soit une isométrie infinitésimale de $\left(V,\langle,\rangle_{\mid V \times V}\right)$. Faisons l'hypothèse que $L_{a} \in B \backslash\{0\}$. Alors il existe $x \in V$ tel que $L_{a}(x) \neq 0$. Supposons que $L_{a}^{k}(x) \neq 0$. Il suit alors que

$$
\left\langle L_{a}^{2 k}(x), x\right\rangle=\left\langle L_{a}^{k}(x), L_{a}^{k}(x)\right\rangle \neq 0
$$

Par conséquent, $L_{a}$ ne peut pas être nilpotent. Donc $B=\{0\}$.

Supposons la représentation $L$ fidèle. On a alors $\operatorname{dim}\left\{L_{a}, a \in A\right\}=n+1$ et comme $B$ est réduite à zéro, on a $\operatorname{dim} A+\operatorname{dim} o(n) \leq \operatorname{dim} o(n, 1)$, ce qui est absurde.

Il reste alors à vérifier que pour $n=1$ ou 2, c'est-à-dire si $A$ est de dimension 2 ou 3 , on a toujours $N(A) \neq\{0\}$. Si $A$ est de dimension 2, le résultat est immédiat puisqu'alors $A$ est abélienne et donc le produit de Levi-Civita est nul. Si $A$ est non abélienne de dimension 3 , alors $A$ est l'algèbre de Lie d'Heisenberg, c'est-à-dire qu'il existe une base $\left\{e_{0}, e_{1}, e_{2}\right\}$ de $A$ telle que $e_{0} \in Z(A)$ et $\left[e_{1}, e_{2}\right]=e_{0}$. Supposons que $L_{e_{0}} \neq 0$. Alors il existe $x, y \in A$ tels que $\left\langle L_{e_{0}} x, y\right\rangle=-(1 / 2)\left\langle[x, y], e_{0}\right\rangle \neq 0$.

Par conséquent $\left\langle e_{0}, e_{0}\right\rangle \neq 0$ et $A=I \oplus I^{\perp}$, où $I=\boldsymbol{R} e_{0}$. Soit $e \in I$ tel que $\langle e, e\rangle= \pm 1$. Puisque $[A, A]=I$, nous avons d'après la formule (4) $\langle x \cdot y, z\rangle=0$ pour tous $x, y, z$ dans 
$I^{\perp}$. Par ailleurs, $\langle x \cdot y, e\rangle=-\langle y, x \cdot e\rangle$, donc le produit symétrique à gauche sur $A$ s'écrit

$$
\begin{aligned}
x \cdot y & =-\langle y, u(x)\rangle e, \\
x \cdot e=e \cdot x & =\lambda(x) e+u(x), \\
e \cdot e & =0,
\end{aligned}
$$

où $\lambda \in\left(I^{\perp}\right)^{*}$ et $u \in g l\left(I^{\perp}\right)$. L'égalité $\langle e \cdot x, y\rangle+\langle x, e \cdot y\rangle=0$ équivaut à $\langle u(x), y\rangle+$ $\langle x, u(y)\rangle=0$. On a de plus $\left[L_{e}, L_{x}\right](e)=0$ donc

$$
e \cdot(\lambda(x) e+u(x))=0,
$$

c'est-à-dire $u^{2}=0$. Par conséquent, le noyau de $u$ est non nul, ce qui est absurde puisqu'alors le centre de $A$ serait de dimension supérieure ou égale à 2 . Donc $L_{e_{0}}=0$.

La connexion de Levi-Civita associée à une métrique bi-invariante (pour laquelle les translations à droite sont des isométries) est donnée par $\nabla_{X} Y=(1 / 2)[X, Y]$ d'aprés la formule (4). Elle est donc plate si et seulement si le groupe en question est 2-nilpotent. Plus généralement, la proposition suivante va nous permettre de décrire (Proposition 3.3) les algèbres de Lie des groupes de Lie pseudo-riemanniens plats où les translations à droite sont aussi des applications affines. Dans ce cas, nous dirons que la connexion de Levi-Civita est bi-invariante.

Proposition 1.2. Soit $(G, g)$ un groupe de Lie simplement connexe pseudoriemannien plat tel que la connexion de Levi-Civita associée à g soit bi-invariante. Alors $G$ est un groupe nilpotent qui contient des sous-groupes à un paramètre non triviaux formés de translations.

DÉmonstration. Il est connu qu'un groupe de Lie $G$ est muni d'une connexion plate bi-invariante si et seulement si le crochet de Lie dans $A=L(G)$ est sous-jacent à un produit associatif (voir par exemple [8]). Par conséquent, si $G$ est un groupe de Lie pseudoriemannien plat dont la connexion de Levi-Civita est bi-invariante, son algèbre de Lie $A$ est munie d'une forme bilinéaire symétrique non dégénérée $\langle$,$\rangle et d'un produit associatif L_{a} b=$ $a \cdot b$ tel que pour tout $a$ dans $A$, l'endomorphisme $L_{a}$ soit antisymétrique. L'égalité $L_{a} \circ L_{b}=$ $L_{a \cdot b}$ implique que $L_{a} \circ L_{b}$ est antisymétrique. Par conséquent, $L_{a} \circ L_{b}+L_{b} \circ L_{a}=0$ pour tous $a, b$ dans $A$, ou ce qui revient au même $L_{a} \circ L_{a}=0$ pour tout $a$ dans $A$. On en déduit que l'algèbre associative $A$ n'a pas d'élément idempotent $e$, c'est-à-dire d'élément non nul $e$ tel que $e \cdot e=e$, car sinon $\left(L_{e} \circ L_{e}\right)(e)=e \neq 0$. Or, l'absence d'idempotent dans une algèbre associative équivaut au fait que cette algèbre soit nilpotente ([1], Th.II.8), c'est à dire au fait qu'il existe $k \geq 2$ tel que

$$
A^{k}=\operatorname{Vect}\left\{x_{1} \cdot x_{2} \cdots x_{k} ; x_{i} \in A\right\}=0 .
$$

Par conséquent, pour $k_{0}$ tel que $A^{k_{0}}=0$ et $A^{k_{0}-1} \neq 0$, on a $L_{a}=0$ pour tout $a \in A^{k_{0}-1} \backslash\{0\}$, donc $G$ contient des sous-groupes non triviaux à un paramètre formés de translations. Par suite, l'algèbre de Lie $A$ est nilpotente. 
2. Le cotangent pseudo-riemannien plat d'un groupe de Lie affine. Analysons en détail l'algèbre de Lie d'un groupe de Lie $G$ pseudo-riemannien plat contenant un sousgroupe distingué abélien totalement isotrope de dimension la moitié. Ceci signifie que $A$ est munie d'une pseudo-métrique plate $\langle$, $\rangle$ pour laquelle il existe un idéal de Lie $I$ abélien tel que $I=I^{\perp}$.

Etudions la structure de l'algèbre symétrique à gauche $(A, \cdot)$, où $L_{a} b=a \cdot b$ désigne le produit de Levi-Civita associé à $\langle$, $\rangle$. De la formule

$$
\left\langle L_{x} a, b\right\rangle=(1 / 2)\{\langle[x, a], b\rangle-\langle[a, b], x\rangle+\langle[b, x], a\rangle\}=0
$$

si $x \in A$ et $a, b \in I$, on déduit que $I$ est un idéal bilatère de $A$, et de

$$
\left\langle L_{a} b, x\right\rangle=-\left\langle b, L_{a} x\right\rangle=0,
$$

que $I$ est à produit nul.

On a alors la suite exacte d'algèbres symétriques à gauche

$$
0 \rightarrow I \rightarrow A \stackrel{\pi}{\rightarrow} A / I=B \rightarrow 0,
$$

où $I$ est à produit nul. Si $s$ est une section linéaire de $\pi$, l'espace vectoriel $s(B)$ est muni d'une structure d'algèbre S.G, définie par $s(a) * s(b)=p(s(a) \cdot s(b))$, où $p$ est la projection sur $s(B)$ parallèlement à $I$, isomorphe à celle de $B$. Dans la suite, nous identifierons donc les algèbres symétriques à gauche $B$ et $s(B)$.

Le produit sur $A$ est alors défini par une structure de $B$-bimodule sur $I$ et un 2-cocycle d'algèbre symétrique à gauche $f: B \times B \rightarrow I$ (voir [13]).

L'égalité $I=I^{\perp}$ signifie que $I$ s'identifie à $B^{*}$ par l'isomorphisme d'espaces vectoriels $\varphi: I \rightarrow B^{*},(x \mapsto\langle x, \cdot\rangle)$. Etudions l'action à gauche de $B$ sur $I=B^{*}$. Pour tous $b, b^{\prime} \in B$ et tous $\beta \in B^{*}$, on a $\left\langle b \cdot \beta, b^{\prime}\right\rangle+\left\langle\beta, b b^{\prime}\right\rangle=0$, c'est-à-dire $\left\langle b \cdot \beta, b^{\prime}\right\rangle=-\left\langle\beta, b b^{\prime}\right\rangle$. Par conséquent,

$$
b \cdot \beta=-{ }^{t} L_{b} \beta,
$$

où $L_{b} b^{\prime}=b b^{\prime}$ désigne la multiplication gauche dans $B$. L'action à gauche de $B$ sur $B^{*}$ est donc la contragrédiente de la représentation $L$.

Etudions l'action à droite de $B \operatorname{sur} B^{*}$. L'application

$$
\begin{aligned}
\Phi_{a, b}: B^{*} & \rightarrow \boldsymbol{R} \\
\beta & \mapsto\langle\beta \cdot a, b\rangle,
\end{aligned}
$$

pour $a, b$ dans $B$, définit sur $B$ un produit noté o par

$$
\langle\beta, a \circ b\rangle=\langle\beta \cdot a, b\rangle,
$$

pour tout $\beta \in B^{*}$.

Nous obtenons ainsi une structure de $B$-bimodule sur $B^{*}$ si et seulement si le produit S.G. sur $B$ et le produit o sont liés par la relation

$$
a \circ(b \circ c)+a(b \circ c)=(a b) \circ c+b \circ(a c)
$$

pour tous $a, b, c$ dans $B$. 
Notons $L^{\prime}$ la représentation linéaire de $B$ dans $B$

$$
\begin{aligned}
& B \rightarrow g l\left(B^{*}\right) \\
& a \mapsto L_{a}^{\prime}: b \mapsto a \circ b
\end{aligned}
$$

Le produit sur $A$ s'exprime alors par

$$
(\alpha+a)(\beta+b)=(\alpha \cdot b+a \cdot \beta+f(a, b))+a b,
$$

où

$$
\left\{\begin{array}{l}
\alpha \cdot b=\left(b^{\prime} \mapsto \alpha\left(b \circ b^{\prime}\right)\right)={ }^{t} L_{b}^{\prime}(\alpha), \\
a \cdot \beta=\left(b^{\prime} \mapsto-\beta\left(a b^{\prime}\right)\right)=-{ }^{t} L_{a} \beta=L_{a}^{*} \beta .
\end{array}\right.
$$

Pour que ce produit soit antisymétrique par rapport à $\langle$,$\rangle il faut et il suffit que le produit \circ$ soit anticommutatif, autrement dit $a \circ b+b \circ a=0$, et que le 2-cocycle $f$ vérifie

$$
f(a, b)(c)+f(a, c)(b)=0
$$

pour tous $a, b, c$ dans $B$.

En résumé nous avons la proposition suivante.

Proposition 2.1. Soit $(A,\langle\rangle$,$) une algèbre pseudo-métrique plate admettant un$ idéal bilatère I totalement isotrope avec $\operatorname{dim} I=(1 / 2) \operatorname{dim} A$. La suite exacte canonique d'algèbres symétriques à gauche

$$
0 \rightarrow I \rightarrow A \rightarrow A / I=B \rightarrow 0
$$

détermine sur l'algèbre $B=A / I$ un produit anticommutatif o vérifiant (5) et munit $B^{*}$ de la structure de B-bimodule (7). L'algèbre symétrique à gauche A est alors extension de $B$ par $B^{*}$ suivant un 2-cocycle $f \in Z_{S G}^{2}\left(B, B^{*}\right)$ vérifiant (8).

Réciproquement soit $B$ est une algèbre symétrique à gauche munie d'un produit anticommutatif o tel que l'égalité (5) soit vérifiée. Alors $B^{*}$ est un B-bimodule pour (7). Si de plus $f \in Z_{S G}^{2}\left(B, B^{*}\right)$ vérifie (8), l'espace vectoriel $A=B^{*} \oplus B$ muni du produit (6) et de la pseudo-métrique définie pour $\alpha, \beta \in B^{*}$ et $a, b \in B$,

$$
\langle\alpha+a, \beta+b\rangle=\alpha(b)+\beta(a)
$$

est une algèbre de Lie pseudo-métrique plate.

Nous poserons

DÉFINITION 2.1. L'algèbre pseudo-métrique plate de Proposition 2.1 est appelée algèbre pseudo-métrique plate cotangente de l'algèbre symétrique à gauche $B$ suivant le produit o et le cocycle $f$.

Si o et $f$ sont nuls, on dira que $A$ est l'algèbre cotangente classique de $B$.

ObSERVATion. Soit $G$ un groupe de Lie simplement connexe dont l'algèbre de Lie $A$ est sous-jacente à un produit S.G. défini par une représentation $L: A \rightarrow g l(A)$. La variété cotangente $T^{*} G$ est munie d'une structure de groupe de Lie dont l'algèbre de Lie $L\left(T^{*} G\right)$ est l'algèbre produit semi-direct de $A$ par $A^{*}$ suivant la représentation contragrédiente de $L$ 
([10]). Le groupe $T^{*} G$ sera dit le cotangent classique du groupe affine $G$. Plus généralement, la structure de groupe de Lie sur $T^{*} G$ dont l'algèbre de Lie est la cotangente de $A$ suivant un produit o et un cocycle $f$ sera dite la structure cotangente tordue du groupe affine $G$. Comme application immédiate de Proposition 2.1, nous avons le résultat suivant.

THÉORÈME 2.1. Soit $G$ un groupe de Lie simplement connexe. Le groupe G admet une métrique pseudo-riemannienne plate invariante à gauche pour laquelle $G$ contient un sous-groupe distingué abélien lagrangien si et seulement si $G$ est isomorphe à un groupe cotangent tordu d'un groupe de Lie affine $H$.

Etudions la complétude des groupes pseudo-riemanniens plats qui sont des cotangents d'un groupe affine. Il suffit de voir à quelles conditions l'algèbre pseudo-métrique plate cotangente d'une algèbre S.G. est munie d'un produit symétrique à gauche nilpotent, c'est-à-dire pour lequel les multiplications droites sont nilpotentes.

Soit $A=B^{*} \oplus B$ l'algèbre pseudo-métrique plate cotangente de $B$ suivant le produit $\circ$ et le 2-cocycle $f$. Les multiplications droites dans $A$ sont données, pour $a, b$ dans $B$ et $\alpha, \beta$ dans $B^{*}$, par

$$
\begin{aligned}
& R_{a} b=b \cdot a=f(a, b)+b a, \\
& R_{a} \beta=\beta \cdot a=(b \mapsto \beta(a \circ b))={ }^{t} L_{a}^{\prime} \beta, \\
& R_{\beta} a=a \cdot \beta=-{ }^{t} L_{a} \beta, \\
& R_{\beta} \alpha=0
\end{aligned}
$$

donc les $R_{\beta}$, sont nilpotents et les $R_{a}$ sont nilpotents si et seulement si les multiplications droites dans $B$ sont nilpotentes ainsi que les endomorphismes ${ }^{t} L_{b}^{\prime}$ de $B^{*}$. On en déduit le lemme suivant.

Lemme 2.1. Soit A l'algèbre pseudo-métrique plate cotangente d'une algèbre symétrique à gauche $B$ suivant le produit o et le 2-cocycle $f$. Alors l'algèbre symétrique à gauche A est nilpotente si et seulement si l'algèbre symétrique à gauche B est nilpotente et le produit $\circ$ est tel que $L_{a}^{\prime}:(b \mapsto a \circ b)$ est nilpotent pour tout a dans $B$.

En particulier l'algèbre cotangente classique d'une algèbre symétrique à gauche $B$ est nilpotente si et seulement si $B$ est nilpotente. Autrement dit, la structure affine du groupe de Lie cotangent classique d'un groupe affine est complète si et seulement si celle du groupe affine de départ est complète.

3. Double extension d'une algèbre de Lie pseudo-métrique plate par une droite. Rappelons quelques faits utiles de la cohomologie des algèbres symétriques à gauche, développée dans [13]. Soit $A$ une algèbre S.G. et $M$ un espace vectoriel. Munir $M$ d'une structure de $A$-bimodule équivaut à se donner deux applications

$$
\begin{aligned}
& \lambda: A \times M \rightarrow M \quad \text { et } \rho: M \times A \rightarrow M \\
& (a, m) \mapsto a_{M} \cdot m \quad(m, a) \mapsto m \cdot a_{M}
\end{aligned}
$$


telles que pour tous $a, b \in A$ et $m$ dans $M$, on ait

$$
\begin{aligned}
& a_{M} \cdot\left(b_{M} \cdot m\right)-b_{M} \cdot\left(a_{M} \cdot m\right)=[a, b]_{M} \cdot m, \\
& a_{M} \cdot\left(m \cdot b_{M}\right)-\left(a_{M} \cdot m\right) \cdot b_{M}=m \cdot(a b)_{M}-\left(m \cdot a_{M}\right) \cdot b_{M} .
\end{aligned}
$$

Si $M$ est un $A$-bimodule, un 2-cocycle de $A$ à valeurs dans $M$ est une application bilinéaire $f: A \times A \rightarrow M$ qui vérifie pour $a, b, c$ dans $A$,

$$
\begin{aligned}
f(a \cdot b-b \cdot a, c)= & f(a, b \cdot c)-f(b, a \cdot c)+a_{M} \cdot f(b, c) \\
& -b_{M} \cdot f(a, c)-(f(a, b)-f(b, a)) \cdot c_{M} .
\end{aligned}
$$

On dit que $f$ est un 2-cobord s'il existe une application linéaire $\varphi: A \rightarrow M$ telle que, pour $a, b$ dans $A$,

$$
f(a, b)=a_{M} \cdot \varphi(b)+\varphi(a) \cdot b_{M}-\varphi(a b) .
$$

On note $H_{S G}^{2}(A, \boldsymbol{R})$ le quotient de l'espace des 2-cocycles $Z_{S G}^{2}(A, \boldsymbol{R})$ par l'espace des 2-cobords $B_{S G}^{2}(A, \boldsymbol{R})$.

Soit $A^{-}$l'algèbre de Lie sous-jacente à $A$ et $N$ un $A^{-}$-module. Un 1-cocycle de $A^{-}$à valeurs dans $N$ est une application linéaire $u: A \rightarrow N$ telle que

$$
u([a, b])=a_{N} \cdot u(b)-b_{N} \cdot u(a) .
$$

On dit que $u$ est un 1-cobord s'il existe $n_{0}$ appartenant à $N$ tel que $u(a)=a_{N} \cdot n_{0}$, pour tout $a$ appartenant à $A$. Nous noterons $H_{L}^{1}(A, N)$ le quotient de l'espace des 1-cocycles $Z_{L}^{1}(A, N)$ par l'espace des 1-cobords $B_{L}^{1}(A, N)$. On a alors le lemme suivant.

LEMME 3.1. Si $(A,\langle\rangle$,$) est une algèbre de Lie pseudo-métrique plate, la formule$ $f(a, b)=\langle u(a), b\rangle$ induit un isomorphisme entre $H_{S G}^{2}(A, \boldsymbol{R})$ et $H_{L}^{1}(A, A)$, où $\boldsymbol{R}$ est vu comme un A-bimodule trivial et $A$ est un A-module pour la représentation $L$.

DÉmonstration. Pour prouver le lemme, il suffit de remarquer que $f$ est un 2cocycle d'algèbre S.G (resp. un 2-cobord) si et seulement si $u$ est un 1-cocycle (resp. un 1-cobord) d'algèbres de Lie.

Donnons maintenant une description des algèbres de Lie pseudo-métriques plates admettant un idéal bilatère $I$ de dimension 1 tel que $I \subset I^{\perp}$ avec $I^{\perp}$ un idéal bilatère.

LEMME 3.2. Soit $(A,\langle\rangle$,$) une algèbre de Lie pseudo-métrique plate. Si I est un idéal$ bilatère de dimension 1 totalement isotrope, alors

1. I est à produit nul, $I \cdot I^{\perp}=0$ et $I^{\perp}$ est un idéal à gauche,

2. $I^{\perp}$ est un idéal à droite $\Leftrightarrow I^{\perp} \cdot I=0$,

3. Si $I^{\perp}$ est un idéal bilatère de A, alors les suites canoniques

$$
\begin{gathered}
0 \rightarrow I \rightarrow I^{\perp} \rightarrow I^{\perp} / I \rightarrow 0, \\
0 \rightarrow I^{\perp} \rightarrow A \rightarrow A / I^{\perp} \rightarrow 0, \\
0 \rightarrow I \rightarrow A \rightarrow A / I \rightarrow 0, \\
0 \rightarrow I^{\perp} / I \rightarrow A / I \rightarrow A / I^{\perp} \rightarrow 0
\end{gathered}
$$


sont des suites exactes d'algèbres symétriques à gauche. L'algèbre de Lie quotient $B=I^{\perp} / I$ admet une pseudo-métrique plate canonique, et (10) et (12) sont des suites exactes scindées d'algèbres de Lie.

DÉmonstration. 1. Pour $x$ dans $I, y$ dans $I^{\perp}$ et $a$ dans $A$, l'égalité

$$
\langle x \cdot y, a\rangle=-\langle y, x \cdot a\rangle=0
$$

entraîne $I \cdot I^{\perp}=0$, donc en particulier $I \cdot I=0$, et

$$
\langle a \cdot y, x\rangle=-\langle y, a \cdot x\rangle=0
$$

entraîne $A \cdot I^{\perp} \subset I^{\perp}$.

2. Pour $x, y$ et $a$ comme dans 1), on a $\langle y \cdot a, x\rangle=0$ si et seulement si $\langle a, y \cdot x\rangle=0$, ce qui montre l'équivalence

$$
I^{\perp} \cdot A \subset I^{\perp} \Longleftrightarrow I^{\perp} \cdot I=0 .
$$

3. Comme $\langle$,$\rangle induit sur I^{\perp}$ une forme bilinéaire de radical $I$, on en déduit que $\langle,\rangle_{\mid I^{\perp} \times I^{\perp}}$ définit, par passage au quotient, une forme bilinéaire non dégénérée sur $B=I^{\perp} / I$, que l'on note $\langle,\rangle_{B}$.

Si l'on note $\bar{x}$ la classe modulo $I$ d'un élément $x \in I^{\perp}$, et $\bar{x} \bar{y}$ le produit symétrique à gauche $\operatorname{sur} B$ on aura $\langle\bar{x}, \bar{y}\rangle_{B}:=\langle x, y\rangle$ et donc, pour $x, y, z$ dans $I^{\perp}$,

$$
\langle\bar{x} \bar{y}, \bar{z}\rangle_{B}+\langle\bar{y}, \bar{x} \bar{z}\rangle_{B}=\langle\bar{x} \cdot y, \bar{z}\rangle_{B}+\langle\bar{y}, \bar{x} \cdot z\rangle_{B}=\langle x \cdot y, z\rangle+\langle y, x \cdot z\rangle=0
$$

donc $\left(B,\langle,\rangle_{B}\right)$ est une algèbre pseudo-métrique plate. De plus, $A / I^{\perp}$ étant de dimension 1 , les suites (10) et (12) sont des suites exactes scindées d'algèbres de Lie.

L'algèbre de Lie pseudo-métrique plate $B=I^{\perp} / I$ sera dite algèbre de Lie pseudométrique plate réduite de $I^{\perp}$ suivant $I$.

Dans la suite, on suppose que $I^{\perp}$ est un idéal bilatère. Posons $I=\boldsymbol{R} e$. Soit $\boldsymbol{R} d$ une droite de $A$ en dualité avec $I$ relativement à $\langle$,$\rangle où d$ est tel que $\langle e, d\rangle=1$. Soit $\hat{B}:=$ $(\text { Vect }\{e, d\})^{\perp}$. Alors $A$ s'identifie en tant qu'espace vectoriel à $\boldsymbol{R} e \oplus \hat{B} \oplus \boldsymbol{R} d$ et $I^{\perp}$ à $\boldsymbol{R} e \oplus \hat{B}$.

Le produit sur $I^{\perp}$ peut s'écrire

$$
(\lambda e+a) \cdot(\mu e+b)=f(a, b) e+a * b,
$$

pour $\lambda, \mu \in \boldsymbol{R}$ et $a, b$ dans $\hat{B}$, où $a * b$ désigne la composante de $a \cdot b$ sur $\hat{B}$. Que ce produit est symétrique à gauche équivaut à dire que l'application $(a, b) \mapsto a * b$ fait de $\hat{B}$ une algèbre S.G. et que $f$ est un 2-cocycle scalaire de cette algèbre.

De plus, l'isomorphisme canonique d'espaces vectoriels $\varphi: \hat{B} \rightarrow B=I^{\perp} / I$, qui à $a$ associe $\bar{a}$ est en fait un isomorphismed'algèbres symétriques à gauche.

Dans la suite, nous identifierons donc les algèbres symétriques à gauche $\hat{B}$ et $B=I^{\perp} / I$, et nous noterons $a b=L_{a}^{\prime} b$ le produit sur $B$.

La suite (9) équivaut ainsi à la donnée de la classe de cohomologie d'un 2-cocycle $f \in$ $Z_{S G}^{2}(B, \boldsymbol{K})$, donc d'un 1-cocycle $u \in Z_{L}^{1}(B, B)$ tel que

$$
f(a, b)=\langle u(a), b\rangle,
$$


pour $a, b$ dans $B$.

Comme $I=\boldsymbol{R} e$ et $I^{\perp}=\boldsymbol{R} e \oplus B$ sont des idéaux bilatères de $A$, donc de Lie, le crochet sur $A$ s'exprime, pour $a, b$ dans $B$, par

$$
\begin{aligned}
& {[d, e]=\mu e,} \\
& {[d, a]=D(a)+\alpha(a) e,} \\
& {[a, b]=[a, b]_{B}+\left\langle\left(u-u^{*}\right)(a), b\right\rangle e,}
\end{aligned}
$$

où $\mu \in \boldsymbol{R}, D \in g l(B)$ et $\alpha \in B^{*}$.

De plus, l'égalité $\langle a \cdot b, d\rangle=\langle u(a), b\rangle$ implique que $D-u \in o\left(B,\langle,\rangle_{B}\right)$, c'est-à-dire $(D-u)^{*}=-(D-u)$.

En utilisant l'égalité (4) qui définit le produit sur $A$, on obtient les formules

$$
\left\{\begin{array}{l}
e \cdot e=e \cdot a=a \cdot e=0, \\
a \cdot b=\langle u(a), b\rangle_{B} e+a b, \\
e \cdot d=0, \\
d \cdot e=\mu e, \\
d \cdot d=b_{0}-\mu d, \\
d \cdot a=-\left\langle b_{0}, a\right\rangle_{B} e+(D-u)(a), \\
a \cdot d=-u(a) .
\end{array}\right.
$$

Ceci définit un produit symétrique à gauche sur $A$ si et seulement si l'on a, pour $a, b$ dans $B$,

$$
(d \cdot a) \cdot b-(a \cdot d) \cdot b=d \cdot(a \cdot b)-a \cdot(d \cdot b),
$$

ce qui équivaut aux égalités (14) et (15) suivantes.

$$
\begin{gathered}
\left\langle b_{0}, a b\right\rangle_{B}=\left\langle\mu u(a)-u^{2}(a)+D(u(a))-u(D(a)), b\right\rangle_{B}, \\
a u(b)-u(a b)=D(a) b+a D(b)-D(a b) .
\end{gathered}
$$

La formule (14) signifie que la forme bilinéaire sur $B$

$$
\omega_{D, u}:(a, b) \mapsto\left\langle\mu u(a)-u^{2}(a)+D(u(a))-u(D(a)), b\right\rangle_{B}
$$

est un 2-cobord scalaire de l'algèbre symétrique à gauche $B$, et la formule (15) veut dire que l'application bilinéaire sur $B$ définie par $\alpha_{u}(a, b)=a u(b)-u(a b)$ est la différentielle de $D$ pour la cohomologie de l'algèbre symétrique à gauche $B$ à valeurs dans le $B$-bimodule canonique $B$.

Remarquons que (15) et $u \in Z_{L}^{1}(B, B)$ entraînent que $D$ est une dérivation de Lie de $B$. Pour résumer, énonçons la proposition suivante.

Proposition 3.1. Soit $(A,\langle\rangle$,$) une algèbre de Lie pseudo-métrique plate et I un$ idéal bilatère de dimension 1 totalement isotrope tel que $I^{\perp}$ soit aussi un idéal bilatère de A. Désignons par $B=I^{\perp} / I$ l'algèbre de Lie pseudo-métrique plate réduite. Alors le produit symétrique à gauche sur A est donné par les formules (13), où u est un 1-cocycle d'algèbre de Lie de $B$ à valeurs dans $B$ vu comme B-module pour la multiplication gauche, et $D$ est 
une dérivation de l'algèbre de Lie $B$, avec $D$ et u liés par (15) et tels que $\omega_{D, u} \in B_{S G}^{2}(B, \boldsymbol{R})$ et $(D-u)$ soit antisymétrique par rappport à la pseudo-métrique $\langle,\rangle_{B}$ sur $B$.

Réciproquement, l'analyse faite ci-dessus fournit une méthode de construction d'algèbres pseudo-métriques plates.

Proposition 3.2. Soit $\left(B,\langle,\rangle_{B}\right)$ une algèbre de Lie pseudo-métrique plate, et soient $u \in Z_{L}^{1}(B, B)$ et $D \in \operatorname{Der}(B)$ liés par (15) tels que $(D-u) \in o\left(B,\langle,\rangle_{B}\right)$ et $\omega_{D, u} \in$ $B_{S G}^{2}(B, \boldsymbol{R})$. Si $b_{0}$ vérifie $\omega_{D, u}(a, b)=\left\langle b_{0}, a b\right\rangle$, alors l'espace vectoriel $A=\boldsymbol{R} e \oplus B \oplus \boldsymbol{R} d$ muni du produit symétrique à gauche (13) et de la pseudo-métrique $\langle$,$\rangle qui étend \langle,\rangle_{B}$ et pour laquelle $\operatorname{Vect}(e, d)$ est un plan hyperbolique orthogonal à B, est une algèbre de Lie pseudo-métrique plate.

Ce qui précède justifie la définition qui suit.

DÉFINITION 3.1. L'algèbre de Lie $(A,\langle\rangle$,$) obtenue dans la proposition (3.2) est ap-$ pelée algèbre pseudo-métrique plate double extension de l'algèbre de Lie pseudo-métrique plate $\left(B,\langle,\rangle_{B}\right)$ suivant $(u, D, \mu)$ et $b_{0}$

Nous avons le corollaire immédiat suivant.

COROllaire 3.1. Soit G un groupe de Lie pseudo-riemannien plat dont l'algèbre de Lie s'obtient par double extension par une droite à partir d'une algèbre de Lie pseudométrique plate. Alors $G$ contient des sous-groupes à un paramètre non triviaux formés de translations.

De plus, Proposition 1.2 nous permet d'établir le résultat qui suit.

Proposition 3.3. Soit $(A,\langle\rangle$,$) une algèbre de Lie pseudo-métrique plate pour$ laquelle le produit de Levi-Civita est associatif. Alors A s'obtient à partir de $\{0\}$ ou à partir de $(\boldsymbol{R} x,\langle x, x\rangle=1)$ par une suite de doubles extensions par une droite.

DÉmonstration. Soit $(A,\langle\rangle$,$) une algèbre de Lie pseudo-métrique plate pour laque-$ lle le produit de Levi-Civita $L_{a} b=a \cdot b$ est associatif. D'aprés Proposition 1.2, l'algèbre de Lie $A$ est nilpotente et $N(A) \neq 0$. Par conséquent $N(A) \cap Z(A)$, où $Z(A)$ désigne le centre de l'algèbre de Lie $A$, est non réduit à zéro. Si $e \in N(A) \cap Z(A) \backslash\{0\}$ alors $I=\boldsymbol{R} e$ et $I^{\perp}$ sont des idéaux bilatères. On a en effet

$$
\begin{aligned}
& \langle e, x \cdot a\rangle=\langle x \cdot e, a\rangle=0, \\
& \langle e, a \cdot x\rangle=-\langle a \cdot e, x\rangle=0
\end{aligned}
$$

pour $a$ dans $I^{\perp}$ et $x$ dans $A$. Donc $A$ s'obtient par double extension à partir d'une algèbre pseudo-métrique plate $\left(B,\langle,\rangle_{B}\right)$. Sur $A=\boldsymbol{R} e \oplus B \oplus \boldsymbol{R} d$, le produit de Levi-Civita est donné par les formules (13) et on constate que l'associativité de ce produit implique l'associativité du produit de Levi-Civita sur $B$. Par conséquent, $A$ s'obtient par une suite de doubles extensions par une droite à partir de $\{0\}$ si $A$ est de dimension paire, ou de $(\boldsymbol{R} x,\langle x, x\rangle=1)$ si $A$ est de dimension impaire. 
La proposition suivante décrit les groupes de Lie nilpotents à métrique lorentzienne plate. On appellera algèbre de Lie euclidienne une algèbre de Lie munie d'une forme quadratique définie positive ou définie négative.

THÉORÈme 3.1. Soit $G$ un groupe de Lie nilpotent. Alors $G$ admet une métrique lorentzienne plate si et seulement si son algèbre de Lie est soit abélienne soit double extension d'une algèbre de Lie euclidienne abélienne par une droite suivant $u=D, \mu=0$ et $b_{0}$, avec $D^{2}=0$ et $\operatorname{Im} D \subset\left(\boldsymbol{R} b_{0}\right)^{\perp}$.

DÉmonstration. Rappelons qu'on a, d'aprés Lemme 1.1, le résultat suivant. Si $(A,\langle\rangle$,$) est nilpotente lorentzienne plate, alors N(A) \neq\{0\}$.

Soit $(A,\langle\rangle$,$) une algèbre de Lie nilpotente lorentzienne plate. Quitte à étudier \left(A,\langle,\rangle^{\prime}\right)$, où $\langle a, b\rangle^{\prime}=-\langle a, b\rangle$ pour tous $a, b$ dans $A$, on peut supposer que $\langle$,$\rangle est de signature$ $(n-1,1)$.

Alors il existe $e \in N(A) \cap Z(A)$ tel que $\langle e, e\rangle$ est négatif ou nul. En effet, si pour tout $0 \neq x \in N(A) \cap Z(A)$, on a $\langle x, x\rangle$ strictement positif, alors l'espace vectoriel sous-jacent à $A$ est somme directe orthogonale de $N(A) \cap Z(A)$ et de $B=(N(A) \cap Z(A))^{\perp}$, et $B$ est alors une sous-algèbre de Lie de $A$, munie d'une métrique lorentzienne plate. Or, ceci est absurde $\operatorname{car} N(B) \cap Z(B)=\{0\}$.

$\mathrm{Si}\langle e, e\rangle$ est strictement négatif, le sous-espace $B=(\boldsymbol{R} e)^{\perp}$ est un idéal bilatère puisqu'on a pour $a, b$ dans $B$,

$$
\langle a \cdot b, e\rangle=-\langle b, a \cdot e\rangle=0 \quad \text { et } \quad a \cdot e=e \cdot a=0 .
$$

Il est clair que la restriction $\langle,\rangle_{B}$ de $\langle$,$\rangle à B \times B$ est définie positive, car si $\left\{e_{2}, \ldots, e_{n}\right\}$ est une base orthogonale de $B$ telle qu'il existe $j \in\{2, \ldots, n\}$ avec $\left\langle e_{j}, e_{j}\right\rangle$ strictement négatif, alors la forme $\langle$,$\rangle est d'indice \geq 2$. Par suite, $\left(B,\langle,\rangle_{B}\right)$ est une algèbre de Lie nilpotente euclidienne plate, donc abélienne d'aprés Théorème 1.2.

Dans le cas où $\langle e, e\rangle$ est nul, l'algèbre de Lie nilpotente $B=(\boldsymbol{R} e)^{\perp} / \boldsymbol{R} e$ est munie, par restriction de $\langle$,$\rangle à (\boldsymbol{R} e)^{\perp} \times(\boldsymbol{R} e)^{\perp}$ puis par passage au quotient, d'une métrique euclidienne plate $\langle,\rangle_{B}$. Par suite, $B$ est abélienne. La droite $I=\boldsymbol{R} e$ est un idéal bilatère, et $I^{\perp}$ est aussi un idéal bilatère. L'algèbre de Lie pseudo-métrique plate $A$ est donc double extension par une droite de $B$ suivant $u, D, \mu$ et $b_{0}$, où $u, D$ sont des endomorphismes de $B$ tels que $D-u \in o\left(B,\langle,\rangle_{B}\right)$, avec $\mu \in \boldsymbol{R}$ tel que

$$
\mu u-u^{2}+[D, u]=0
$$

et $b_{0} \in B$. Autrement dit, l'espace vectoriel sous-jacent à $A$ se décompose suivant $A=$ $\boldsymbol{R} \boldsymbol{e} \oplus B \oplus \boldsymbol{R} d$, le crochet de Lie de $A$ étant donné par

$$
\begin{aligned}
& {[d, e]=\mu e,} \\
& {[d, a]=D(a)-\left\langle b_{0}, a\right\rangle_{B} e,} \\
& {[a, b]=\left\langle\left(u-u^{*}\right)(a), b\right\rangle_{B} e,}
\end{aligned}
$$


où $a, b \in B$. Puisque $A$ est nilpotente, on a $\mu=0$, et il existe $k_{0} \in N$ tel que $\left(\operatorname{ad}_{d}\right)^{k_{0}}=0$. Or, pour tout $k$ dans $N$, on a pour $a \in B$,

$$
\left(\operatorname{ad}_{d}\right)^{k}(a)=D^{k}(a)-\left\langle b_{0}, D^{k-1}(a)\right\rangle e .
$$

En effet, c'est vrai pour $k=1$, et si c'est vrai au rang $k$, on a pour $a \in B$,

$$
\left(\operatorname{ad}_{d}\right)^{k+1}(a)=\left[d,\left(\operatorname{ad}_{d}\right)^{k}(a)\right]=\left[d, D^{k}(a)\right]=D^{k+1}(a)-\left\langle b_{0}, D^{k}(a)\right\rangle_{B} e .
$$

Donc si $A$ est nilpotente d'ordre $k_{0}$, c'est-à-dire $C^{k_{0}}(A)=0$ et $C^{k_{0}-1}(A) \neq 0$, alors $D$ est nilpotent d'ordre $k_{0}$ et $\operatorname{Im} D^{k_{0}-1} \subset\left(\boldsymbol{R} b_{0}\right)^{\perp_{\langle,\rangle_{B}}}$. Réciproquement, si $D$ est nilpotent d'ordre $k_{0}$ et $\operatorname{Im} D^{k_{0}-1} \subset\left(\boldsymbol{R} b_{0}\right)^{\perp_{\langle,\rangle_{B}}}$, alors $A$ est nilpotente car $e \in Z(A)$ et $\left(\operatorname{a} d_{a}\right)^{2}=0$ pour $a$ dans $B$. La formule $u^{2}=[D, u]$ implique alors que pour tout $k \geq 1$,

$$
(D-u)^{k}=D^{k}-k u \circ D^{k-1},
$$

donc $D-u$ est nilpotent. Comme $\langle,\rangle_{B}$ est euclidienne et $D-u \in o\left(B,\langle,\rangle_{B}\right)$, on a donc $D=u$. Par conséquent, $D^{2}=u^{2}=[D, u]=0$, et $A$ est au plus nilpotente d'ordre 3 .

4. Exemples. Commençons par mettre en évidence quelques groupes de Lie où toute métrique pseudo-riemannienne invariante est non plate.

Proposition 4.1. Le seul groupe de Lie d'Heisenberg qui admet une métrique pseudo-riemannienne plate invariante est celui de dimension 3.

DÉmonstration. Le groupe de Lie d'Heisenberg de dimension 3 admet une métrique lorentzienneplate invariante, puisque son algèbre de Lie $A$ est double extension de $(B=$ $\left.\boldsymbol{R} b,\langle b, b\rangle_{B}=1\right)$ par une droite suivant $u=D=0, \mu=0$ et $b_{0}=b$.

Supposons que $\langle$,$\rangle est une pseudo-métrique plate sur A=L\left(H_{2 k+1}\right)$, avec $k>1$. Il en résulte que le noyau de l'algèbre symétrique à gauche $A$ est non réduit à zéro. En effet, soit $e$ un générateur de $Z(A)=[A, A]$. Alors $\langle e, e\rangle=0$, sinon $A=\boldsymbol{R} e \oplus(\boldsymbol{R} e)^{\perp}$, avec $x \cdot y=-\langle u(x), y\rangle e$ et $e x=x e=\lambda(x) e+\langle e, e\rangle u(x)$, pour tous $x, y$ dans $(\boldsymbol{R} e)^{\perp}$, où $u$ est antisymétrique et $u^{2}=0$. Or, ceci est absurde car le centre de $A$ serait de dimension strictement supérieure à 1 . Puisque $[A, A]=\boldsymbol{R} e$ et pour tous $x, y$ dans $A,\langle e \cdot x, y\rangle=$ $-(1 / 2)\langle[x, y], e\rangle$, on a $L_{e}=0$.

Ceci entraîne que $A$ s'obtient par double extension à partir d'une algèbre pseudo-métrique plate $\left(B,\langle,\rangle_{B}\right)$ suivant $u \in \circ\left(B,\langle,\rangle_{B}\right), D=0, b_{0}$ et $\mu=0$, avec $u 2$-nilpotent. Mais ceci implique que le noyau de $u$ est de dimension supérieure à 1 , ce qui est impossible car $Z(A)$ est de dimension 1.

Mutatis mutandis, on obtient un résultat analogue à celui de Murakami (voir [14], p. 213) dont nous utiliserons la propriété suivante.

Proposition 4.2. Soit $M$ une variété différentiable munie d'une connexion localement plate $\nabla$ et d'une métrique pseudo-riemannienne $g$.

Les conditions suivantes sont équivalentes:

1. La pseudo-métrique $g$ est localement hessienne relativement à $\nabla$. 
2. $\left(\nabla_{Z} g\right)(X, Y)=\left(\nabla_{Y} g\right)(X, Z)$ pour tous champs de vecteurs $X, Y, Z$ sur $M$.

Nous pouvons énoncer alors le résultat suivant.

THÉORÈME 4.1. Le groupe de Lie $G=G L(n, \boldsymbol{R})$ n'admet pas en général de métrique pseudo-riemannienne plate invariante. Cependant il admet des pseudo-métriques biinvariantes localement hessiennes relatives à la structure affine bi-invariante canonique.

DÉmonstration. D'après Proposition 4.2, dire qu'un groupe de Lie admet une métrique localement hessienne invariante équivaut à dire que son algèbre de Lie $\mathcal{G}$ admet une forme quadratique non dégénérée $\langle$,$\rangle telle que, pour tous x, y$ et $z \in \mathcal{G}$, on a

$$
\langle x \cdot y, z\rangle-\langle y \cdot x, z\rangle=\langle x, y \cdot z\rangle-\langle y, x \cdot z\rangle,
$$

où $(x, y) \mapsto x \cdot y$ est le produit symétrique à gauche sur $\mathcal{G}$ dont provient la structure de Lie de $\mathcal{G}$ (voir [15]).

Soit $\langle$,$\rangle la forme bilinéaire symétrique non dégénérée définie sur \mathcal{G}=L(G)$ par

$$
\langle M, N\rangle=\operatorname{tr}(M N),
$$

pour tous $M, N$ dans $\mathcal{G}=M_{n}(\boldsymbol{R})$. L'égalité $\langle M N, P\rangle=\langle M, N P\rangle$ pour tous $M, N, P$ dans $\mathcal{G}$ prouve que la métrique pseudo-riemannienne invariante sur $G$ qui coïncide avec $\langle$, en l'élément neutre est bi-invariante et localement hessienne relativement à la structure affine définie par le produit des matrices (voir [3]).

Montrons que le groupe de Lie $G=G L(2, \boldsymbol{R})$ n'admet pas de métrique pseudoriemannienne plate invariante.

Supposons que $G$ soit un groupe de Lie pseudo-riemannien plat. Son algèbre de Lie $\mathcal{G}=M_{2}(\boldsymbol{R})$ admet alors une forme quadratique non dégénérée $q$ et les formules (1), (2) et (3) entraînent que $\varphi: \mathcal{G} \rightarrow \circ(\mathcal{G}, q),\left(x \mapsto L_{x}\right)$ est une représentation d'algèbres de Lie telle que $\operatorname{Id}_{\mathcal{G}}$ soit un 1-cocycle, c'est-à-dire

$$
\varphi(x) y-\varphi(y) x=[x, y],
$$

pour tous $x, y$ dans $\mathcal{G}$. Puisque $\mathcal{G}^{\prime}=\operatorname{sl}(2, \boldsymbol{R})$ est semi-simple, la restriction de $\varphi$ à $\mathcal{G}^{\prime}$ est complètement réductible. Il se présente alors trois cas. Soit $\mathcal{G}$ est un $\mathcal{G}^{\prime}$-module simple, soit $\mathcal{G}$ se décompose en somme directe de deux $\mathcal{G}^{\prime}$-modules simples de dimension 2, soit $\mathcal{G}$ se décompose en somme directe d'un $\mathcal{G}^{\prime}$-module simple de dimension 1 et d'un $\mathcal{G}^{\prime}$-module simple de dimension 3.

Soit $\{H, X, Y, T\}$ la base de $\mathcal{G}$ définie par

$$
H=\left(\begin{array}{cc}
1 & 0 \\
0 & -1
\end{array}\right), \quad X=\left(\begin{array}{ll}
0 & 1 \\
0 & 0
\end{array}\right), \quad Y=\left(\begin{array}{ll}
0 & 0 \\
1 & 0
\end{array}\right), \quad T=\left(\begin{array}{ll}
1 & 0 \\
0 & 1
\end{array}\right) .
$$

La description des modules irréductibles sur $\operatorname{sl}(2, \boldsymbol{R})([7])$, nous permet de voir qu' il n'existe pas de représentation de $\mathcal{G}$ par des isométries infinitésimales de $(\mathcal{G}, q)$ telle que $\mathcal{G}$ soit un $\mathcal{G}^{\prime}$-module simple. En effet, dans ce cas il existe une base $\mathcal{B}=\left(e_{1}, e_{2}, e_{3}, e_{4}\right)$ de $\mathcal{G}$ telle que $\varphi(H)\left(e_{1}\right)=3 e_{1}, \varphi(H)\left(e_{2}\right)=e_{2}, \varphi(H)\left(e_{3}\right)=-e_{3}, \varphi(H)\left(e_{4}\right)=-3 e_{4}$ et telle que 
$\varphi(X)\left(e_{1}\right)=0, \varphi(X)\left(e_{2}\right)=3 e_{1}, \varphi(X)\left(e_{3}\right)=2 e_{2}, \varphi(X)\left(e_{4}\right)=e_{3}$. Soit $\langle$,$\rangle la forme po-$ laire associée à $q$. Le fait que $\varphi(H) \in \circ(\mathcal{G}, q)$ implique alors que $\left\langle e_{1}, e_{3}\right\rangle=\left\langle e_{3}, e_{3}\right\rangle=$ $\left\langle e_{4}, e_{3}\right\rangle=0$, car les espaces propres associés à des valeurs propres non opposées sont orthogonaux relativement à $q$. Mais comme l'égalité $\left\langle\varphi(X)\left(e_{3}\right), e_{3}\right\rangle=0$ entraîne que $\left\langle e_{2}, e_{3}\right\rangle=0$, ceci est impossible.

Supposons maintenant que $\mathcal{G}$ se décompose en somme directe de deux $\mathcal{G}^{\prime}$-modules simples de dimension 2. Dans ce cas, il existe une base $\mathcal{B}=\left(e_{1}, e_{2}, e_{3}, e_{4}\right)$ de $\mathcal{G}$ telle que $\varphi(H)\left(e_{1}\right)=e_{1}, \varphi(H)\left(e_{2}\right)=-e_{2}, \varphi(H)\left(e_{3}\right)=e_{3}, \varphi(H)\left(e_{4}\right)=-e_{4}, \varphi(X)\left(e_{1}\right)=\varphi(X)\left(e_{3}\right)=$ $0, \varphi(X)\left(e_{2}\right)=e_{1}, \varphi(X)\left(e_{4}\right)=e_{3}$ et $\varphi(Y)\left(e_{1}\right)=e_{2}, \varphi(Y)\left(e_{2}\right)=\varphi(Y)\left(e_{4}\right)=0, \varphi(Y)\left(e_{3}\right)=$ $e_{4}$. La matrice de $q$ dans la base $\mathcal{B}$ s'écrit alors

$$
\left(\begin{array}{cccc}
0 & 0 & 0 & \beta \\
0 & 0 & -\beta & 0 \\
0 & -\beta & 0 & 0 \\
\beta & 0 & 0 & 0
\end{array}\right) .
$$

Comme $\varphi(T)$ commute avec $\varphi(x)$ pour tout $x$ dans $\mathcal{G}$ et est antisymétrique par rapport à $q$, sa matrice dans la base $\mathcal{B}$ est de la forme

$$
\left(\begin{array}{cccc}
a & 0 & c & 0 \\
0 & a & 0 & c \\
b & 0 & -a & 0 \\
0 & b & 0 & -a
\end{array}\right) .
$$

On constate alors que Id n'est pas un 1-cocycle pour $\varphi$ car la condition (16) ne peut pas être vérifiée. Dans le cas où $\mathcal{G}$ se décompose en somme directe d'un $\mathcal{G}^{\prime}$-module simple de dimension 1 et d'un $\mathcal{G}^{\prime}$-module simple de dimension 3 , la même démarche montre que Id n'est pas un 1-cocycle.

4.1. Groupes de Lie pseudo-riemanniens plats de dimension 2 et 3. Toute métrique pseudo-riemannienne invariante sur un groupe de Lie abélien étant plate, on s'intéresse dans la suite à l'existence de métriques pseudo-riemanniennes plates invariantes sur un groupe de Lie non abélien.

4.1.1. Dimension 2. Le groupe affine de la droite $G A(\boldsymbol{R})$. D'après le résultat de Milnor, ce groupe n'admet pas de métrique plate invariante. Par contre, on peut le voir comme le groupe cotangent pseudo-riemannien plat classique d'un groupe affine de dimension 1, ou comme le groupe pseudo-riemannien plat double extension du groupe réduit à zéro par une droite. La pseudo-métrique plate obtenue sur $\mathcal{G}=A_{2}=\operatorname{Vect}\{a, b\}$, où $[a, b]=b$, est celle pour laquelle $\langle a, a\rangle=\langle b, b\rangle=0$ et $\langle a, b\rangle=1$.

4.1.2. Les groupes de Lie pseudo-riemanniens plats de dimension 3. Le résultat précédent permet de définir sur le groupe produit direct du groupe affine de la droite par une droite une métrique lorentzienne plate invariante, produit de la métrique hyperbolique plate invariante sur $G A(\boldsymbol{R})$ et d'une métrique invariante sur la droite.

Il est connu qu'un groupe semi-simple n'admet pas de structure affine, donc les autre groupes de dimension 3 qui admettent une pseudo-métrique plate sont nécessairement les 
produits semi-directs d'un groupe de dimension 1 par un groupe distingué abélien. Soit donc $d$ un endomorphisme de $B=\boldsymbol{R}^{2}$ et $A$ l'algèbre produit semi-direct de $\boldsymbol{R} d$ par $B$, de crochet de Lie $[d, x]=d(x)$, pour tout $x$ dans $B$.

Si $A$ admet une pseudo-métrique plate $\langle$,$\rangle telle que B$ soit dégénéré, alors $A$ est double extension d'une algèbre de Dimension $1,\left(\boldsymbol{R} b,\langle b, b\rangle^{\prime}=1\right)$ par une droite suivant $u=$ $(1 / 2)\left(D+D^{*}\right), D, \mu$ et $b_{0}$, où $D$ vérifie $\mu\left(D+D^{*}\right)-(1 / 2)\left(D+D^{*}\right)^{2}=0$, et ceci signifie que $d$ est nilpotent (dans ce cas $A$ est l'algèbre de Lie du groupe d'Heisenberg), ou bien a une valeur propre double.

Si $A$ admet une pseudo-métrique plate $\langle$,$\rangle telle que B$ soit non dégénéré, alors $d$ a soit deux valeurs propres opposées, soit son polynôme minimal est de la forme $\mu(X)=X^{2}+\alpha^{2}$.

Le tableau suivant fournit les pseudo-métriques plates ainsi mises en évidence sur les algèbres de Lie non abéliennes de dimension 3 .

\begin{tabular}{|c|c|c|}
\hline $\mathrm{A}$ & crochet de Lie & pseudo-métrique plate \\
\hline$A_{2} \times \boldsymbol{R}$ & {$\left[e_{1}, e_{2}\right]=e_{2}, e_{3} \in Z(A)$} & $\left(\begin{array}{lll}0 & 1 & 0 \\
1 & 0 & 0 \\
0 & 0 & 1\end{array}\right)$ \\
\hline \multirow{2}{*}{$L\left(H_{3}\right)$} & {$\left[e_{1}, e_{2}\right]=e_{3}, e_{3} \in Z(A)$} & $\left(\begin{array}{lll}0 & 0 & 1 \\
0 & 1 & 0 \\
1 & 0 & 0\end{array}\right)$ \\
\hline \multirow{2}{*}{$B \times \boldsymbol{R} d_{\alpha}$} & {$\left[e_{3}, e_{1}\right]=\alpha e_{1},\left[e_{3}, e_{2}\right]=\alpha e_{2}$} & $\left(\begin{array}{lll}0 & 0 & 1 \\
0 & 1 & 0 \\
1 & 0 & 0\end{array}\right)$ \\
\hline \multirow{2}{*}{$B \times \boldsymbol{R} d_{\alpha}$} & {$\left[e_{3}, e_{1}\right]=\alpha e_{1},\left[e_{3}, e_{2}\right]=e_{1}+\alpha e_{2}$} & $\left(\begin{array}{lll}0 & 0 & 1 \\
0 & 1 & 0 \\
1 & 0 & 0\end{array}\right)$ \\
\hline \multirow{2}{*}{$B \times \boldsymbol{R} d_{\alpha}$} & {$\left[e_{3}, e_{1}\right]=\alpha e_{1},\left[e_{3}, e_{2}\right]=-\alpha e_{2}$} & $\left(\begin{array}{lll}0 & 1 & 0 \\
1 & 0 & 0 \\
0 & 0 & 1\end{array}\right)$ \\
\hline \multirow{2}{*}{$B \times \boldsymbol{R} d_{\alpha}$} & {$\left[e_{3}, e_{1}\right]=\alpha e_{2},\left[e_{3}, e_{2}\right]=-\alpha e_{1}$} & $\left(\begin{array}{lll}1 & 0 & 0 \\
0 & 1 & 0 \\
0 & 0 & 1\end{array}\right)$ \\
\hline
\end{tabular}

où le crochet et la pseudo-métrique s'exprime dans la même base $\left(e_{1}, e_{2}, e_{3}\right)$.

4.2. D'autres exemples de groupes de Lie pseudo-riemanniens plats. Soit $B=\boldsymbol{R}^{n}$ l'algèbre de Lie abélienne de dimension $n$ et $d$ un endomorphisme de $B$. Notons $A$ l'algèbre de Lie $B \oplus \boldsymbol{R} d$ produit semi-direct de $\boldsymbol{R} d$ par $B$ suivant $d$, c'est-à-dire dont le crochet de Lie est donné pour tout $x$ dans $B$, par $[d, x]=d(x)$. Si nous munissons $B$ d'une forme bilinéaire symétrique non dégénérée $\langle,\rangle_{B}$ telle que $d$ soit un endomorphisme antisymétrique de l'espace quadratique $\left(B,\langle,\rangle_{B}\right)$, la pseudo-métrique $\langle$,$\rangle sur A$ qui étend $\langle,\rangle_{B}$ et telle que $\boldsymbol{R} d$ soit orthogonal à $\left(B,\langle,\rangle_{B}\right)$ est plate. En particulier, si $d$ est un endomorphisme nilpotent 
principal $\left(d^{n}=0, d^{n-1} 0\right)$, on peut construire une telle pseudo-métrique si et seulement si $n$ est impair. Dans ce cas il suffit de poser $\left\langle e_{i}, e_{n-i+1}\right\rangle_{B}=(-1)^{i+1}$, pour $1 \leq i \leq n$, où $\left(e_{1}, \ldots, e_{n}\right)$ est une base de $B$ telle que $d\left(e_{1}\right)=0$ et $d\left(e_{k}\right)=e_{k-1}$ pour $1<k \leq n$. Si $d$ est diagonalisable, il faut et il suffit que ses valeurs propres non nulles soient deux à deux opposées. On peut alors définir $\langle,\rangle_{B}$ par

$$
\begin{aligned}
\left\langle e_{2 i-1}, e_{2 i}\right\rangle=1 & \text { pour } \quad 1 \leq i \leq k, \\
\left\langle e_{j}, e_{j}\right\rangle=1 \quad \text { pour } \quad 2 k+1 \leq j \leq n, & \text { si } i \neq j, \quad \text { si } i \neq j-1, \quad \text { ou si } i \neq j+1,
\end{aligned}
$$

où $\left(e_{1}, \ldots, e_{n}\right)$ est une base formée de vecteurs propres de $B$ telle que pour tout $1 \leq i \leq k$, les vecteurs $e_{2 i-1}$ et $e_{2 i}$ soient associés à des valeurs propres opposées, non nulles, et les autre vecteurs de base appartiennent au noyau de $d$.

Dans le cas où $d$ est nilpotent principal mais où $n$ est pair, $A$ admet aussi une pseudométrique plate car elle s'obtient par double extension: elle est alors somme orthogonale du plan hyperbolique abélien Vect $\left\{e_{1}, d\right\}$ et de l'algèbre abélienne Vect $\left\{e_{2}, \ldots, e_{n}\right\}$ munie de la pseudo-métrique définie par $\left\langle e_{i}, e_{n-i+2}\right\rangle=(-1)^{i}$ pour $2 \leq i \leq n-1$.

En résumé, nous avons la proposition suivante.

PROPOSITION 4.3. Les algèbres de Lie produit semi-direct d'une droite par une algèbre de Lie abélienne $B=\boldsymbol{R}^{n}$ suivant $d \in \operatorname{End}(B)$ admettent une pseudo-métrique plate

(i) si d est nilpotent principal $\left(d^{n}=0, d^{n-1} \neq 0\right)$,

(ii) si d est diagonalisable et ses valeurs propres non nulles sont deux à deux opposées.

\section{RÉFERÉNCES}

[1] A. Albert, Structure of Algebras, Amer. Math. Soc. Colloq. Publ.24, American Mathematical Society, New York, 1939.

[2 ] A. AUBERT, Thèse de doctorat, Université Montpellier II, 1996.

[ 3 ] M. Bordemann, Invariante Bilinearformen auf endlich-dimensionalen Algebren, Diplomarbeit, Mathematische Fakultät, Universität Freiburg, F.R.G., 1988.

[4] J. M. Dardié And A. Medina, Double-extension symplectique d'un groupe de Lie symplectique, Adv. Math. 117 (1996), 208-227.

[ 5 ] J. Helmstetter, Radical et groupe formel d'une algèbre symétrique à gauche, thèse de doctorat de 3ème cycle, Université de Grenoble, 1975.

[ 6 ] J. Helmstetter, Radical d'une algèbre symétrique à gauche, Ann. Inst. Fourier (Grenoble) 29 (1979), $17-$ 35.

[ 7 ] J. E. Humphreys, Introduction to Lie algebras and representation theory, Graduate Texts in Mathematics 9, Springer-Verlag, NewYork-Berlin, 1972.

[ 8 ] A. Medina, Flat left-invariant connections adapted to the automorphism structure of a Lie group, J. Differential Geom. 16 (1981), 445-474.

[9] A. Medina And P. Revoy, Groupes de Lie à structure symplectique invariante, Symplectic geometry, groupoids, and integrable systems (Berkeley, CA, 1989), 247-266, Math. Sci. Res. Inst. Publ. 20, Springer Verlag, New York, 1991.

[10] A. Medina And P. Revoy, Algèbres de Lie et produit scalaire invariant, Ann. Sci. École Norm. Sup. (4) 18 (1985), 553-561. 
[11] J. Milnor, Curvatures of Left Invariant Metrics On Lie Groups, Adv. in Math. 21 (1976), 293-329.

[12] J. MiLnor, On fundamental group of complete affinely flat manifolds, Adv. in Math. 25 (1977), 178-187.

[13] A. NiJENHUIS, Sur une classe de propriétés communes à quelques types différents d'algèbres, Enseignement Math. (2) 14 (1968), 225-277.

[14] H. SHImA, On certain locally flat homogeneous manifolds of solvable Lie groups, Osaka J. Math. 13 (1976), 213-229.

[15] H. SHIMA, Homogeneous Hessian manifolds, Ann. Inst. Fourier (Grenoble) 30 (1980), 91-128.

DÉPARTEMENT Des MathÉMATiQues, CC. 051

UNIVERSITÉ DE MONTPELLIER II

Place E. Bataillon

34095 MONTPELLIER CEDEX 5

FRANCE

E-mail addresses: aubert@math.univ-montp2.fr medina@math.univ-montp2.fr 